\title{
Impact of Uncertainty on Wind Power Curtailment Estimation
}

\author{
Joseph Dillon \\ Electricity Research Centre, \\ UCD, Ireland \\ jody.dillon@ucd.ie
}

\author{
Mark O’Malley \\ Electricity Research Centre, \\ UCD, Ireland \\ mark.omalley@ucd.ie
}

\begin{abstract}
Ireland and other countries in the EU have binding targets for production of energy from renewable sources by 2020. Ireland's Renewable Energy Action Plan aims to meet this target by producing $40 \%$ of electrical energy from renewable sources and most of this will come from wind power. In order to forecast the amount of wind power capacity required, it is necessary to forecast the amount of wind power curtailment that will arise from the need to maintain a certain amount of conventional generation online to provide system services such as reserve, inertia and system balance. Estimation of future levels of wind power curtailment is also necessary for investors. In this paper, a stochastic scheduling model is used to study the impact of forecast error related uncertainty on wind power curtailment estimation. Results are shown illustrating the impact of uncertainty on final energy production from wind power and the impact improvements in forecasting could have on these estimates.
\end{abstract}

Keywords—variable renewables; wind power; stochastic scheduling;

\section{Introduction}

As the penetration of wind power production increases on systems, the impact of uncertainty becomes more pronounced as does the potential need to curtail wind power output to ensure system integrity. The need for these curtailments is primarily due to the variability and uncertainty associated with wind power combined with the need for system balance, sufficient system services such as inertia and as a result of congestion arising from limitations of the transmission network. On the Irish system the so called, system non-synchronous penetration limit (SNSP) [1] is the manifestation of this need to maintain a minimum amount of conventional generation on the system to ensure system integrity. Curtailments due to operational issues, such as the SNSP constraint and minimum loads, are the focus of this paper. Curtailments due to congestion, while important in other systems, have not been studied here.

Deterministic unit commitment models have often been used to examine the impact of high levels of wind power on the Irish system and to estimate the level of wind power curtailment [2]. These approaches often do not account for uncertainty which has a significant impact on system operation. Some deterministic approaches do make allowance for uncertainty, for example, by dispatching against a point forecast and including reserve constraints to allow for forecast error (so-called robust scheduling), but these approaches tend to overstate the impact due to the use of tools not specifically designed to address uncertainty. The All Island Grid Study [3], [4] featured the utilization of a stochastic model to assess uncertainty. This approach used the WILMAR [4] tool and was based on synthetic wind forecast error data. Based on lessons learned and experience using the WILMAR tool, a flexible stochastic optimization tool called Epiphron has been in development and this has been further developed as part of this study to examine the impacts of uncertainty on the Irish power system in 2020. Methods of managing this uncertainty through the use of stochastic scheduling have been examined and the impact of improved forecasting has been assessed.

Previous work on investigating the impact of wind power uncertainty on wind power curtailment e.g. [5], [6] has used just one set of wind power forecast scenarios based on synthesized data.

In this study the scenario generation functionality of the Epiphron tool was used to generate many sets of stochastic scenarios for wind and for load. The wind scenarios have been based on the statistics of real historical forecast versus realized data and these have been used in the two stage stochastic scheduling component of the tool. This has allowed for a more accurate representation of uncertainty. Since hydro power is exclusively run of river in Ireland, this was modelled deterministically based on monthly historical averages however in systems with large amounts of hydro, this can be an important source of uncertainty.

Section 2 presents the stochastic scheduling model used and the scenario generation methodology. Section 3 presents the test system which the methodology is applied to along with details of the test cases examined. Results are summarized in Section 4. Finally, conclusions arising from the work are presented in Section 5.

\section{Stochastic Scheduling Methodology}

\subsection{General Methodology}

In order to estimate the impact of wind power uncertainty, conventional deterministic production cost models do not suffice in that they typically assume perfect foreknowledge of wind power production. In reality, a system operator must make decisions regarding the commitment of generating units, storage trajectories and interconnector flows based on an imperfect forecast of wind power output. Decisions regarding commitment of generating units are generally made ahead of time and may be difficult to reverse in the short term due to technical restrictions on units such as minimum up and down times, shut down times and notice times. However, unit commitments of fast starting generators can be changed and such flexible conventional generation is of value in managing uncertainty, particularly in systems with large amounts of renewable generation. To 
replicate the process of making decisions under uncertainty, there are two main requirements:

1. A scheduling model that replicates the process of making future commitment decisions of generating plant based on imperfect forecasts. These are known as two-stage scheduling models and consist of a forward looking stage where future strategic decisions are made based on forecasts and a realization stage that simulates the adjustments required in real time to account for actual wind power and load outcomes. The forward looking stage determines the commitment of units, storage trajectories and interconnector flows which are then available in the realization stage.

2. A set of stochastic scenarios based on the characterization of the forecast error which can be used in the forward looking stage of the scheduling algorithm.

\subsection{Scheduling Model and Rolling Planning}

The model implements a stochastic rolling planning approach which attempts to simulate the process of determining future unit commitments, hydro trajectories and interconnector schedules against future uncertain load and wind power production. Real time dispatch decisions must also be made given the unit commitments, hydro trajectories and interconnector schedules determined in previous planning steps. The model seeks to determine future strategic decisions that will give the best chance of satisfying operational constraints and which minimize expected operating costs. Resources are dispatched in real time to meet actual realized values of demand and variable renewable production while future commitments can be re-optimized as new forecast information becomes available. The problem consists of two stages within the same optimization which are solved simultaneously: A realization stage where real time dispatch is determined based on realized values of wind power production and load and where strategic decisions determined in the previous stochastic planning stage, such as unit commitments, are fixed. This is followed by a stochastic stage which determines future strategic decisions based on stochastic scenarios of wind power production and customer demand. Thus, in realization stage $\mathrm{T}$, the commitment variables for slow starting units are fixed to the values determined in the previous stochastic planning stage, $\mathrm{T}-1$. In the stochastic phase, generating units must have the same commitment in each stochastic scenario but may have different dispatch levels. This models the requirement to determine a robust future unit commitment schedule which will be robust against a number of different possible outcomes.

Strategic decisions such interconnector flows, hydro and storage trajectories and unit commitments are fixed across scenarios while shorter term decisions such as unit operating levels, reserve provision and wind power production are variable across scenarios. This simulates the process of taking longer term decisions to maximize the expected benefit in respect of a range of uncertain future outcomes. Subject to inter-temporal limitations imposed by decision in past intervals, these variables are re-optimized each step (every 6 hours in this study but variable in the model).

Since we compare three distinct models in this paper we have chosen not to present the full formulation of each here. The basic unit commitment model follows the formulation presented in [8]. The interesting differences are described in the text.

\subsection{Characterisation of Forecast Error}

In order to characterize the statistical properties of the forecast error, one year of historical forecast vs. actual wind power data was analyzed. This data was segmented based on the look-ahead time of the forecast. The statistical moments of the forecast error distribution were calculated for each look-ahead time. This captures the phenomenon that forecasts for periods in the shorter term are more accurate than for longer term time periods [7]. The first 10 auto-correlations of individual forecasts over time were also calculated. Stochastic scenarios for demand were produced using the same approach but using the statistical moments from [4] rather than from historical data.

\subsection{Scenario Generation}

A moment matching approach was used to generate the stochastic scenarios for the model. This is based on the work in [8] and adapted for the stochastic structure of the present model. The basic idea of this approach is to generate a set of wind and load forecast scenarios that have statistical moments which match those calculated for the actual historical forecast vs. actual data. This approach involves creating initial scenarios based on random draws from a normal distribution. A non-linear programming problem is then formulated to minimize the squared difference between the statistical moments of the simulated scenarios and the corresponding values calculated from actual historical forecast errors. The same is done for the first 6 autocorrelations of the simulated scenarios. The four moments considered in this work are mean, variance, skewness and kurtosis. In addition to a more accurate statistical representation of the forecast error [9], computing performance has also been increased over the autoregressive moving average (ARMA) series approach adopted in [4].

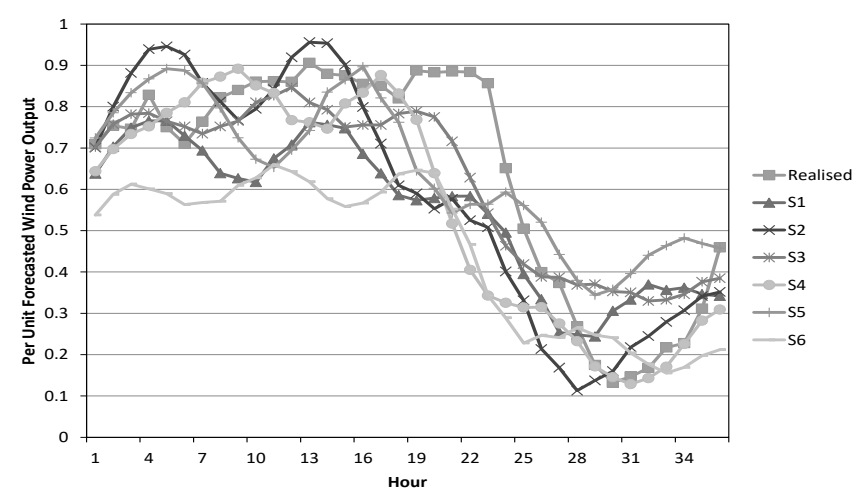

Figure 1. Sample set of wind power forecast scenarios

To generate scenarios of forecasted wind power and demand, allowance must be made for the fact that the expected value of 
forecast error will not be zero. This can be accomplished by adding forecast error to actual historical forecasts to generate forecasted production scenarios which capture this phenomenon. However, this requires coincident production and forecast information with different look-ahead values to be available and this will not generally be the case. In this work, coincident historical forecast data was available for wind power production but not for system demand. The approach adopted here for system demand forecasts was to initially generate two scenarios of forecast error, each with a probability of 0.5 . One of these was arbitrarily chosen and added to the realized system demand to simulate the expected forecast. Six scenarios of forecast error were then produced and each added to the simulated expected forecast to generate six forecasted system demand scenarios. A sample set of wind power forecast scenarios can be seen in Figure 1, above.

\subsection{Synthesizing Forecasting Improvements}

It is expected that by 2020 wind forecasting performance will have improved due to a number of factors:

- Improvements to the numerical weather prediction models on which physical wind forecasting tools ultimately depend

- Wider geographic diversity and the so called "smoothing" effect

- Increased numbers of wind farm forecasts that are included in the system level forecast (system level wind power predictions are normally based on forecasts for a subset of the entire fleet and scaled up to the system level wind power capacity).

- Improvements in software and wind power prediction tools

To simulate the impact of improved forecasting, the stochastic scenarios of wind power forecast which are based on historical actually observed forecasting performance are blended with the actual realized wind power production in the following way:

$$
w_{f n}=a \cdot w_{f o}+(1-a) \cdot w_{r}
$$

where $w_{f n}$ is the new improved forecast value, $w_{f o}$ is the original forecast value, $w_{r}$ is the realized value and $a$ is a value between 0 and 1 indicating the desired level forecast improvement. A value of zero would represent no improvement and a value of 1 would indicate perfect forecasting. Here, improvements to forecasting performance are examined as a sensitivity case. An "improved forecasting" case was constructed by using a value of 0.9 for $a$, in (1). This approximately equates to an improvement of $10 \%$. This approach is simple to implement but is not based on any knowledge of how wind power forecasting may actually improve. Future work will consider how this may better be accomplished. However it is likely that the simplified method adopted here is sufficient to capture the first order benefits of improvement.

\subsection{Pseudo Stability Constraints}

A number of pseudo constraints are included in the model to represent the need for conventional generation for dynamic and frequency stability. These are coarse approximations that represent the impacts of phenomenon that cannot be directly included in the model.

- $\quad$ Based on the results of dynamic and frequency stability studies, generation from non-synchronous sources is currently limited to $55 \%$ with the potential for this limit to increase once a number of mitigation measures have been completed [1]. This is known as the system nonsynchronous penetration limit (SNSP). It is assumed that by the study year (2020) this limit has been raised to $75 \%$.

- At least 5 large conventional units must be kept online in Ireland and 2 in Northern Ireland.

\subsection{Operational Constraints}

Minimum up and down times, minimum stable levels operating reserve, pumped storage operational constraints and run-of-river hydro power energy limits have been modeled and formulated as in [8]. Based on [5], it was assumed that it was sufficient to model Primary Operating Reserve only.

\section{Test System}

The year 2020 was chosen as the study year as Ireland has a binding target for energy from renewable sources. This includes an electricity sector target of $40 \%$ energy from renewable sources with $37 \%$ expected to come from wind power. Transmission congestion has not been modelled in this study as congestion levels are forecasted to be very low for this year and adequate transmission reinforcement is a pre-requisite for new generation on the Irish system. While a single node model is adequate for the test system presented here, it is recognized that locational issues are important for many systems and are a complicating factor in stochastic optimization [10].

Conventional plant data for existing plant is based on published information [11]. Data for new plant has been based on [12]. Assumptions regarding interconnection with Great Britain have also been based on [12]. As other studies have forecasted the level of transmission congestion to be very low for the year 2020, no transmission was modelled in this study.

\subsection{Test Cases}

In this work, three distinct cases have been examined for consideration of forecast error related uncertainty in the operational time frame.

3.1.1 Deterministic with Perfect Foresight. In this scheduling method a conventional unit commitment algorithm is used where no account is taken of forecast error. This is the reference case and represents a lower bound on curtailment. It is assumed that future wind power production is known perfectly 
in advance of making unit commitment decisions. This case has been implemented using the Epiphron tool in conventional deterministic unit commitment mode.

3.1.2 Point Forecasts. Here a two-stage scheduling model is used to synthesize the impact of forecast error related uncertainty. In the forward planning stage future unit commitments, hydro and interconnector schedules are determined based on a point forecast of future wind power production. In the realization stage, the commitment of slow starting units, interconnector flows and hydro trajectories are fixed according to decisions made in the previous planning stage and the system must be re-dispatched to account for differences between the forecasted and realized wind power and load. Both stages are solved simultaneously to ensure continuity between the realization and planning stages. It is possible in this approach that infeasibilities will arise. The objective function includes penalty factors for constraint violations such as demand balance (where the penalty factor equals the value of lost load, or VOLL) but also includes penalties for reserve and ramping constraint violations. New forecasts are assumed to be available every 6 hours and the system is re-optimized to account for new forecast information. This scenario represents the outcomes that would arise if forecast information was used exclusively to base future scheduling decisions upon. This would represent the practice of using point forecasts to dispatch the system and represents an upper bound on curtailment levels due to forecast error related uncertainty. This is equivalent to stochastic scheduling as described below, but with only one scenario.

3.1.3 Stochastic Scheduling. Here scenarios of possible future wind power production and load are generated based on historical forecasts and historical forecast performance. Rolling planning is used as described above and future commitment decisions are based on a range of possible wind power outcomes (rather than just one as in the two-stage deterministic with point forecast scenario). Each possible scenario has a certain probability of occurring related to the statistics of historical forecast error. This allows the system to dispatch conservatively to account for a range of possible future outcomes. Here, uncertainty is represented, but knowledge of the statistics of the forecast error is used to minimize the expected costs and thus curtailment. This would represent future curtailment outcomes if the system operator were using a stochastic unit commitment tool to dispatch the system.

\section{Results}

\subsection{Impact of Uncertainty on Wind Power Curtailment Estimates}

Figure 2 below compares the system level wind curtailment rates for each of the three scheduling models. Curtailment results in the models when full utilization of the available wind power would result in a constraint violation. For example, a constraint described in Section 2.6 requires that, for stability reasons, a minimum number of conventional units must be kept online at any time. Thus, in some periods it may be necessary to reduce wind power below its available production with a corresponding increase in conventional generation. The curtailment results in Figure 2 show that forecast error related uncertainty will result in higher levels of wind power curtailment than that predicted by deterministic perfect foresight models. The results also show that curtailment is significantly reduced by using stochastic scheduling compared to a two-stage model with point forecasts. These results show that when compared to stochastic scheduling, use of conventional deterministic models with perfect foresight result in curtailment estimates which are approximately $40 \%$ lower with $4700 \mathrm{MW}$ of wind capacity and $30 \%$ lower with $5190 \mathrm{MW}$ of wind capacity. This suggests that the presence of uncertainty in real life operations would lead to higher curtailment levels than is predicted by deterministic perfect foresight models. It should be noted that any curtailment of wind power is matched by a corresponding increase in conventional generation of equal magnitude.

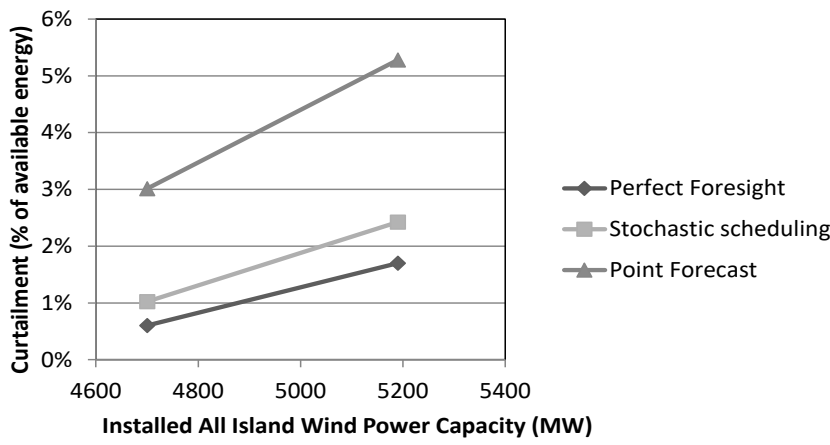

Figure 2. Wind power curtailment for different methods of accounting for uncertainty.

\subsection{Impact of uncertainty on 2020 Targets}

The total percentage of energy from wind power in each case is shown in Figure 3 below along with the amount required to meet the 2020 target. It can be seen that because wind power curtailment is lower in the stochastic scheduling case compared to the deterministic two-stage with point forecast case, less wind power capacity is required to meet the 2020 targets. Table 1, below shows the estimated amount of wind power capacity that would be required to meet Ireland's 37\% wind energy target in each case by linearly interpolating between the data points from Figure 3.

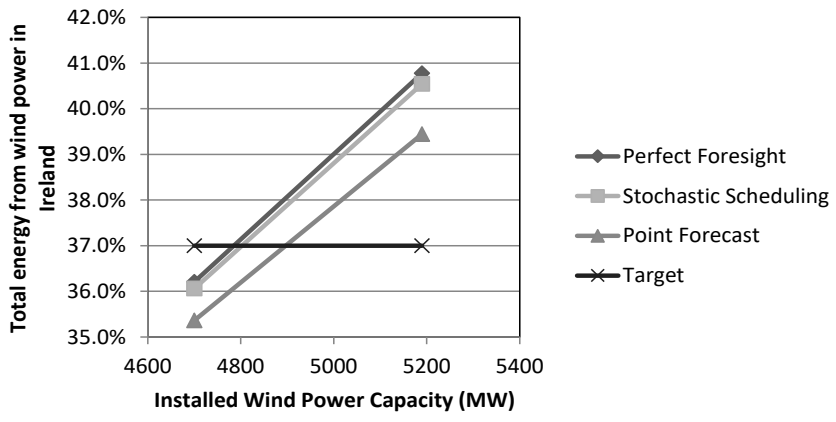

Figure 3. Total energy from wind power for different methods of accounting for uncertainty. 
Table 1. Total estimated wind power capacity required to meet 2020 targets

\begin{tabular}{|c|c|c|c|}
\hline & $\begin{array}{c}\text { Perfect } \\
\text { Foresight }\end{array}$ & $\begin{array}{c}\text { Stochastic } \\
\text { Scheduling }\end{array}$ & $\begin{array}{c}\text { Point } \\
\text { Forecast }\end{array}$ \\
\hline $\begin{array}{c}\text { All Island Wind Capacity } \\
\text { Required to Meet 2020 } \\
\text { Target (MW) }\end{array}$ & 4785 & 4803 & 4897 \\
\hline
\end{tabular}

\subsection{Impact of Forecast Error on Operating Costs}

Table 2, below shows the total operating costs (generator fuel and start costs, emissions costs and taxes) saved by using stochastic scheduling instead of point forecasts. These operating cost savings arise due to reduced wind power curtailment. Reduced curtailment means increased wind power production which displaces conventional generation thus resulting in a cost saving. The results show that better representation of uncertainty result in a reduction of between $1.1 \%$ and $1.6 \%$ of total system operating costs, depending on the level of installed wind power capacity. The saving is considerable and outweighs the relatively small investment required to develop a stochastic optimization capability.

Table 2. Cost savings from using stochastic scheduling

\begin{tabular}{|c|c|c|}
\hline $\begin{array}{c}\text { Installed } \\
\text { Wind Power } \\
\text { (MW) }\end{array}$ & $\begin{array}{c}\text { Saving Using Stochastic } \\
\text { over Point Forecast } \\
\mathbf{\epsilon m}\end{array}$ & $\begin{array}{c}\text { Saving Using } \\
\text { Stochastic over Point } \\
\text { Forecast }\end{array}$ \\
\hline 4700 & 18.7 & $1.1 \%$ \\
\hline 5190 & 26.9 & $1.6 \%$ \\
\hline
\end{tabular}

\subsection{Impact of SNSP on Wind Power Curtailment Estimates}

Table 3. Impact on wind power curtailment of a reduced SNSP level of $65 \%$ compared to the $75 \%$ case.

\begin{tabular}{|c|c|c|c|}
\hline & \multicolumn{3}{|c|}{ Curtailed Energy in the High-Wind Case } \\
\hline SNSP Level & $\begin{array}{c}\text { Perfect } \\
\text { Foresight }\end{array}$ & $\begin{array}{c}\text { Stochastic } \\
\text { Scheduling }\end{array}$ & Point Forecast \\
\hline $75 \%$ & $1.7 \%$ & $2.4 \%$ & $5.3 \%$ \\
\hline $65 \%$ & $4.9 \%$ & $6.4 \%$ & $10.2 \%$ \\
\hline
\end{tabular}

While it is assumed that the SNSP level will reach $75 \%$ by 2020, a sensitivity case was run to examine the impact of a lower SNSP level. Table 3, above shows the impact on curtailment for a lower SNSP limit of $65 \%$. It can be seen that there is a significant increase in curtailment.

\subsection{Impact of Improved Forecasting Performance}

It could be expected that there will be some improvement to forecasting accuracy over the coming years. To capture the impact of such improvements, a set of forecast wind power scenarios were synthesized using the methodology described in sub-section 2.5. Table 4, below shows the reduced curtailment that results when forecasting performance is improved by $10 \%$ and $20 \%$ on average.
Table 4. Curtailment in \% of total available energy for the improved forecasting cases (5190MW of installed wind power)

\begin{tabular}{|c|c|c|}
\hline Case & Stochastic Scheduling & Point Forecast \\
\hline Base & $2.42 \%$ & $5.28 \%$ \\
\hline $10 \%$ Improvement & $2.33 \%$ & $5.17 \%$ \\
\hline $20 \%$ Improvement & $2.21 \%$ & $5.34 \%$ \\
\hline
\end{tabular}

Table 5, below shows the cost reductions for improvements in wind power forecasting accuracy.

Table 5. Cost reductions due to improved forecasting (5190MW of installed wind power)

\begin{tabular}{|c|c|c|}
\hline $\begin{array}{c}\text { Improved Forecasting } \\
\text { Performance }\end{array}$ & Stochastic Scheduling & Point Forecast \\
\hline $10 \%$ Improvement & $€ 6.7 \mathrm{~m}(0.4 \%)$ & $€ 3.5 \mathrm{~m}(0.2 \%)$ \\
\hline $20 \%$ Improvement & $€ 11.5 \mathrm{~m}(0.7 \%)$ & $€ 4.1 \mathrm{~m}(0.2 \%)$ \\
\hline
\end{tabular}

\section{Conclusions and Discussion}

Modelling of forecast error related uncertainty is required to produce accurate levels of curtailment as simple deterministic models significantly underestimate the amount of curtailment that may be required. In energy terms, curtailment is underestimated by $40 \%$ by simple deterministic perfect foresight models compared to stochastic models.

The methodology presented here also demonstrates the cost and renewable energy maximizing benefits of stochastic scheduling. Use of stochastic scheduling has the potential to deliver significant cost savings (our study suggests between $1.1 \%$ and $1.6 \%$ of total system costs or between $€ 19 \mathrm{~m}$ and $€ 27 \mathrm{~m}$ annually, depending on the installed wind power capacity) and can result in significantly reduced curtailment over pointforecasts used in conjunction with conventional scheduling methods

Improved forecasting performance has the potential to reduce system costs and lower curtailment levels.

Delays in transitioning to $75 \%$ SNSP will result in significantly increased curtailment, potentially beyond the key $5 \%$ level which is perceived to be the threshold of commercial viability in the sector.

\section{Acknowledgment}

This work was conducted in the Electricity Research Centre, University College Dublin, Ireland, which is supported by the Commission for Energy Regulation, Bord Gáis Energy, Bord na Móna Energy, Cylon Controls, EirGrid, EPRI, ESB International, ESB Power Generation, ESB Networks, Gaelectric, Intel, Siemens, SSE Renewables, SWS Energy, UTRC and Viridian. This publication has emanated from research conducted with the financial support of Science Foundation Ireland under grant number SFI/09/SRC/E1780. Research conducted with the financial support of Science Foundation Ireland under grant number SFI/09/SRC/E1780. 


\section{References}

[1] O’Sullivan, J., Rogers, A., Flynn, D., Smith, P., Mullane, A., and O'Malley, M.J., "Studying the Maximum Instantaneous NonSynchronous Generation in an Island System - Frequency Stability Challenges in Ireland", IEEE Transactions on Power Systems

[2] Holttinen, H., Orths, A., Abildgaard, H., Van Hulle, H., Kiviluoma, J., Lange, B., O’Malley M., Flynn, D., Keane, A., Dillon, J., Carlini, E. M., Tande, J. O., Estanqueiro, A., Lazaro, E. G., Söder, L., "IEA wind Recommended Practices, Wind Integration Studies", International Energy Agency, $\quad$ Sept 2013. http://www.ieawind.org/Task 25/PDF/HomePagePDF\%27s/RP\%2016 \%20Wind\%20Integration\%20Studies_Approved\%20091213.pdf.

[3] Department of Communications, Energy and Natural Resources, "All Island Electricity Grid Study". Available at: http://www.dcenr.gov.ie/Energy/North-South+Cooperation+in+the+Energy+Sector/All+Island+Electricity+Grid+Study.ht $\underline{\mathrm{m}}$

[4] Meibom, P.; Barth, R., Brand, H.; Hasche, B.; Swider, D.; Ravn, H.; Weber, C., "All Island Grid Study Workstream 2(b): Wind Variability Management Studies" Sustainable Energy, IEEE Transactions on , vol.3, no.4, pp.760,768, Oct. 2012

[5] Tuohy, A.; Meibom, P.; Denny, E.; O'Malley, M., "Unit Commitment for Systems With Significant Wind Penetration," Power Systems, IEEE Transactions on , vol.24, no.2, pp.592,601, May 2009

[6] Meibom, P., Barth, R., Hasche, B., Brand, H., Weber, C. and O’Malley, M.J., "Stochastic optimisation model to study the operational impacts of high wind penetrations in Ireland", IEEE Transactions on Power Systems, Vol. 26, pp. 1367 - 1379, 2011.

[7] Madsen, H., Pinson, P., Kariniotakis, G., Nielsen, H. and Neilsen, T., "Standardizing the Performance Evaluation of Short-Term Wind Power Prediction Models", Wind Engineering Volume 29, No. 6, pp 475-489, 2005

[8] Carrion, M. and Arroyo, J. M., "A computationally efficient mixedinteger linear formulation for the thermal unit commitment problem," in IEEE Transactions on Power Systems, vol. 21, no. 3, pp. 1371-1378, Aug. 2006.

[9] Lowery, C.; O'Malley, M., "Impact of Wind Forecast Error Statistics Upon Unit Commitment," Sustainable Energy, IEEE Transactions on , vol.3, no.4, pp.760,768, Oct. 2012

[10] Lee, C., Liu, C., Mehrotra, S. and Shahidehpour, M. "Modeling Transmission Line Constraints in Two-Stage Robust Unit Commitment Problem," in IEEE Transactions on Power Systems, vol. 29, no. 3, pp. 1221-1231, May 2014.

[11] The All Island Project, "2011 Plexos Validation Reports and Models", http://www.allislandproject.org/en/market_decision_documents.aspx?art icle=151a9561-cef9-47f2-9f48-21f6c62cef34

[12] EirGrid, "All-Island Generation Capacity Statement 2013-2022”, 2013, available online at http://www.eirgrid.com/media/AllIsland GCS 2013-2022.pdf 